\title{
BBXRT OBSERVATIONS OF SUPERNOVA REMNANTS
}

\author{
R. PETRE, P. J. SERLEMITSOS, F. E. MARSHALL, K. JAHODA, E. A. \\ BOLDT, S. S. HOLT, R. F. MUSHOTZKY, J. SWANK, A.E. \\ SZYMKOWIAK, R. KELLEY, A. SMALE, K. ARNAUD, and K. WEAVER \\ Code 666, Laboratory for High Energy Astrophysics \\ NASA / Goddard Space Flight Center \\ Greenbelt, Maryland 20771 \\ USA
}

\begin{abstract}
The BBXRT observed nine supernova remnants during its nine-day flight. We present preliminary results from some of these observations, emphasizing the ability of BBXRT to perform spatially resolved spectroscopy. The improved spectral resolution and efficiency over previous instruments makes possible measurements of previously undetectable lines, and the broad bandpass allows simultaneous measurement of lines from oxygen through iron.
\end{abstract}

\section{Introduction}

During its nine-day flight on the Space Shuttle Astro-1 mission, the Broad Band X-ray Telescope (BBXRT) observed X-ray sources of all types, including stars, X-ray binaries, supernova remnants, active galaxies, clusters of galaxies, and the X-ray background. While its broad bandpass (0.3-12 keV), moderate spectral resolution (155 eV at $6 \mathrm{keV})$, high efficiency, and extremely low background (Serlemitsos et al. 1991) make it an extremely versatile instrument and useful for study of any of these types of objects, the study of supernova remnants using the BBXRT is highly attractive for two additional reasons. First, study of extended objects such as supernova remnants allows utilization of BBXRT's capability for carrying out spatially resolved spectroscopy. As shown in Figure 1 , a map of the Tycho supernova remnant projected onto the BBXRT focal plane, the detector consists of five discrete elements: a central circular detector of radius 2.25 arc minutes, surrounded by an outer annulus to a radius of 8.5 arc minutes divided into four quadrants. All elements are separated by a 1.5 arc minute wide, $X$-ray opaque, mask. The size of these elements and their separation are well matched to the $\sim 3$ arc minutes (HPD) spatial resolution of the X-ray mirrors. Second, the broad bandpass allows for the first time simultaneous observation of $\mathrm{K}$-shell transitions of astrophysically interesting elements from oxygen through nickel, plus the L-shell transitions of iron. The improved spectral resolution over previous broad band spectrometers, as illustrated in Figure 2, makes possible more accurate determination of line properties for plasma diagnostics, as well as measurements of previously undetectable lines. Below we survey the BBXRT observations of supernova remnants and present some preliminary results. While a more detailed analysis awaits a better knowledge of the instrument calibration, many of our results are independent of the details of the calibration, and it some of these that we highlight here. 


\section{Observations}

The BBXRT acquired scientifically useful exposures of approximately 60 sources. Nine of these were supernova remnants. These were the historical remnants Cas A, Tycho, and the Crab; the evolved remnants Puppis A, IC 443, and G109.1-1.0; the region around the Vela pulsar; N132D in the LMC; and SN1987a. Exposures ranged from $475 \mathrm{~s}$ (for N132D) to $3600 \mathrm{~s}$ (for Puppis A). As it is impossible in this brief presentation to discuss all of these, we simply point out the richness of the data collected.

\section{Preliminary Results from Selected Remnants}

\subsection{TYCHO}

Tycho, the remnant of a Type I supernova whose explosion was observed in 1572 , is one of the most extensively studied of all supernova remnants. The current best model of the X-ray spectrum, which used data from a number of instruments to cover the entire X-ray band, invokes a time-dependent ionization model with two components, one representing the propagating blast wave, and the other a reverse shock encountering layered ejecta (Hamilton, Sarazin, and Szymkowiak 1986). With the BBXRT it was possible to observe simultaneously the high and low energy components and thus remove any systematic uncertainties associated with instrumental cross calibrations. The BBXRT pointing location is shown in Figure 1. The central element observed a bright portion of the $\mathrm{X}$-ray shell (the

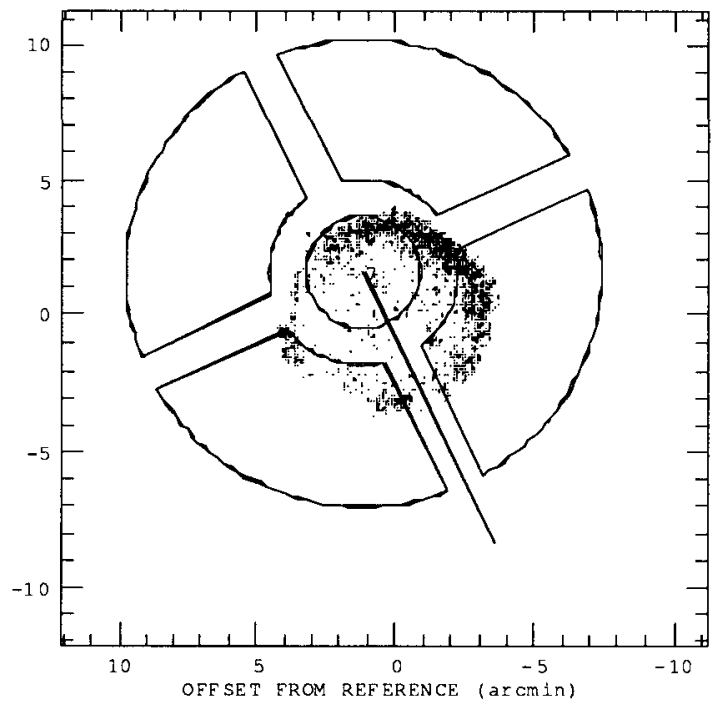

Figure 1: BBXRT observation of Tycho, showing individual detector elements.

spectrum of which is compared in Figure 2 with that from a much longer Einstein SSS exposure); the opposite side of the shell was observed by an outer element. The two $\mathrm{BBXRT}$ spectra are remarkably similar, despite the fact that they represent emission from regions separated by a distance of approximately $6 \mathrm{pc}$. This suggests that the supernova event was symmetric and is expanding into a uniform local interstellar medium. A number of strong lines are visible to the eye, including some that were previously never resolved 

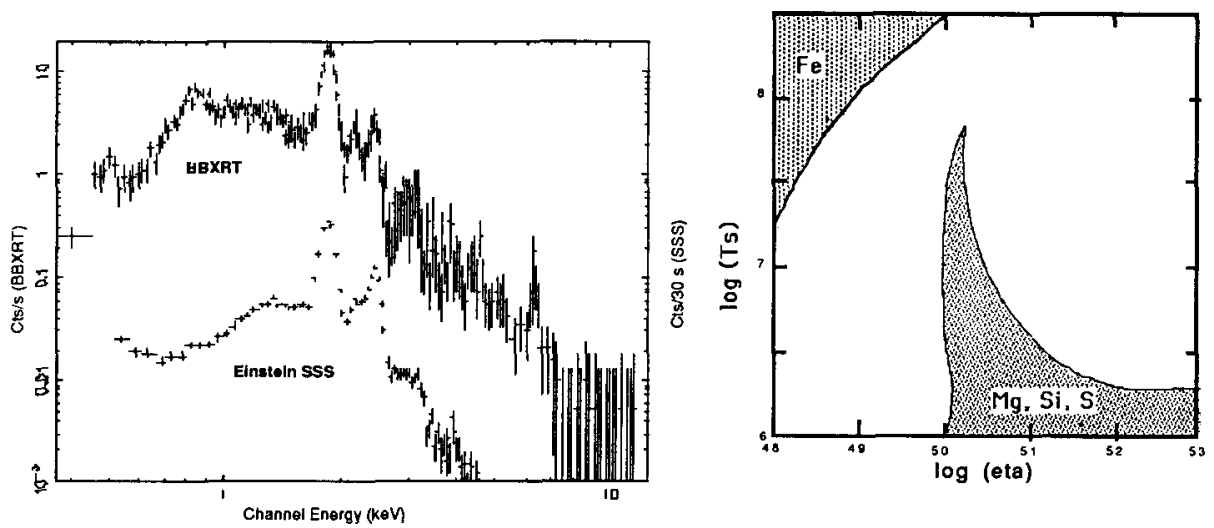

Figure 2 (left): BBXRT spectrum of Tycho, compared with Einstein SSS spectrum. The SSS data have bee rescaled for clarity of illustration. Figure 3 (right): Allowed 90 percent confidence ranges of shock temperature (Ts) and $\eta$ from Tycho line diagnostics.

(for example, the "beta" transition of helium-like silicon at $2.01 \mathrm{keV}$ ). Merely measuring the energy centroids and the intensity ratios of the prominent lines provides some new and interesting information. For instance, one of the sources of controversy based on the results of lower energy spectrometers is the centroid energy of the $\mathrm{Fe} \mathrm{K}$ line (Hamilton, Sarazin, and Szymkowiak 1986, and reference therein). BBXRT measures this centroid directly at $6.41 \pm 0.02 \mathrm{keV}$. Using diagnostics for nonequilibrium plasmas (Hamilton, Sarazin, and Chevalier 1983), we can constrain the parameters characterizing the plasma: $\mathrm{Ts}$, the shock temperature, and $\eta\left(=n^{2} \mathrm{E}\right.$, where $\mathrm{n}$ is the preshock density and $\mathrm{E}$ is the explosion energy). As shown in Figure 3, the parameter values allowed by diagnostics using the lighter elements ( $\mathrm{Mg}, \mathrm{Si}$, and $\mathrm{S}$ ) are disjoint from those allowed by the $\mathrm{Fe} \mathrm{K}$ line energy. We conclude that physical conditions encountered by the Fe, which represents the innermost shell of ejecta, are hotter and less dense than those encountered by outer shells. While the possibility of this was suggested by Hamilton, Sarazin, and Szymkowiak (1986), BBXRT provides a direct measurement almost trivially.

\subsection{G109.1-1.0}

The remnant G109.1-1.0 is unique among galactic supernova remnants: in addition to a well-defined, semicircular shell, it contains a centrally situated binary X-ray pulsar. (Gregory and Fahlman 1980; Fahlman and Gregory 1981) It thus allows us to study whether the presence of such a pulsar influences the evolution of the remnant. The angular extent is just the right size so that BBXRT could simultaneously obtain spectra of both the shell and the pulsar in different detector elements. Figure 4 shows where the instrument was pointed. In Figure 5 we show the strikingly different spectra from the shell and the pulsar. The pulsar spectrum is well fit by a simple power law model with a very steep photon index $(\Gamma=3.9 \pm 0.2)$, consistent with that measured by EXOSAT (Morini et al. 
1988). Spectral features near 1 and $4 \mathrm{keV}$ may also be present. The BBXRT performed the first ever high quality measurement of the shell spectrum, uncontaminated by the pulsar ( 20 percent upper limit on contamination). The spectrum displays prominent lines (Fe $\mathrm{L} ; \mathrm{Mg}$ and $\mathrm{Si}$ K) and is well fit by a two-temperature equilibrium plasma model, which can be used to mimic non-equilibrium plasmas. The appearance of the spectrum suggests that the evolution of at least the observed portion of the shell is essentially unaffected by the pulsar.

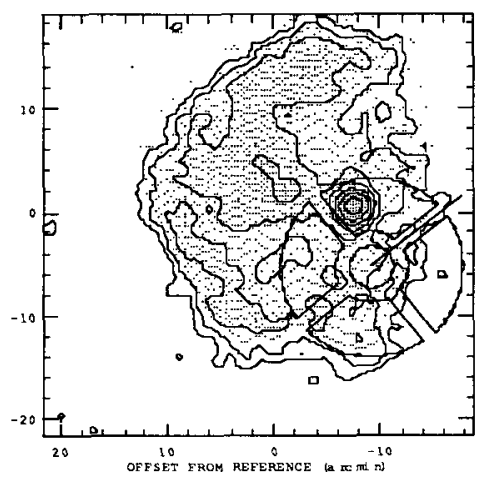

Figure 4 (above): SNR G109.1-1.0 projected onto BBXRT focal plane.
Figure 5 (right): Spectrum of pulsar $1 \mathrm{E} 2259+586$ (top). Spectrum of diffuse emission from shell of G109.1-1.0 (bottom).

\subsection{IC 443}

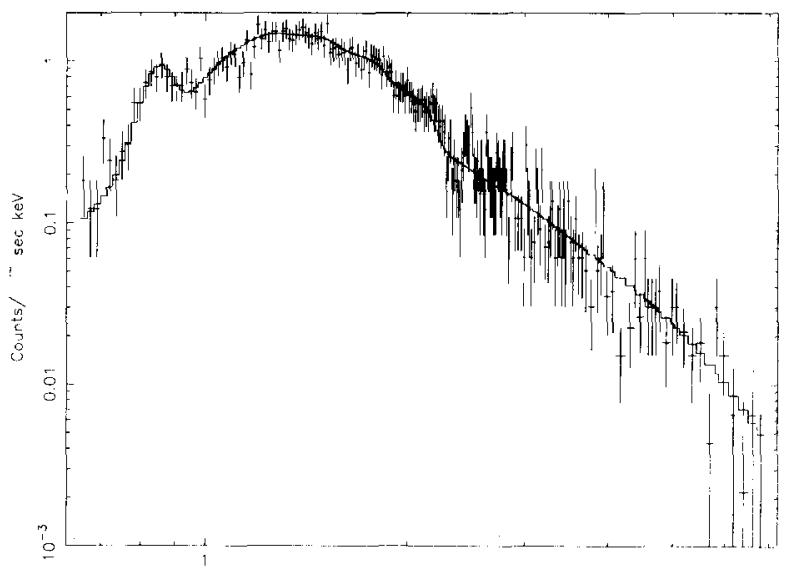

The global X-ray morphology of the evolved remnant IC 443 is strongly influenced by the presence of a molecular cloud in the line of sight (Petre et al. 1988). Bright X-ray emission, relatively unobscured by the cloud, arises in a region of enhanced density in the vicinity of (but not associated with) bright optical filamentation in the northeast of the remnant. The goal of the BBXRT observation was to perform spatially resolved spectroscopy of the bright region, whose 13 arc minute extent is well matched to the 17 arc minute BBXRT field of view. In particular, variations of column density due to the molecular cloud and of temperature and ionization conditions were being sought. In Figure 6 we show spectra from the edge of the shell, and from approximately 7 and 10 arc minutes behind the edge. Spectral differences are quite noticeable. There is a clear trend with increasing distance behind the shell towards lines of higher ionization state in the Fe $L$ band around $1 \mathrm{keV}$, the $\mathrm{Mg} \mathrm{K}$ band around $1.34 \mathrm{keV}$ and the $\mathrm{Si} \mathrm{K}$ band around $1.9 \mathrm{keV}$. 
When using a two-temperature model we find that while the same pair of temperatures $(0.3$ and 1.2 $\mathrm{keV}$ ) fit all three spectra, the ratio of the components changes by a factor of four in favor of the hotter component towards the interior. These results suggest that we are directly observing the upward ionization of the interstellar gas toward ionization equilibrium.

Figure 6: Spectra of bright region of IC 443 , taken at the edge of the shell (top), and approximately 7 arc minutes (middle) and 10 arc minutes (bottom) bchind the shell. Increases in the average energy of the $\mathrm{Fe} \mathrm{L}$ blend near $1 \mathrm{keV}$ and the $\mathrm{Mg} \mathrm{K}$ line near $1.35 \mathrm{keV}$ are readily apparent. Units on vertical scale are counts $/ \mathrm{sec} / \mathrm{keV}$; the absolute scalings of the spectra have been offset for illustration purposes.

\section{Summary}

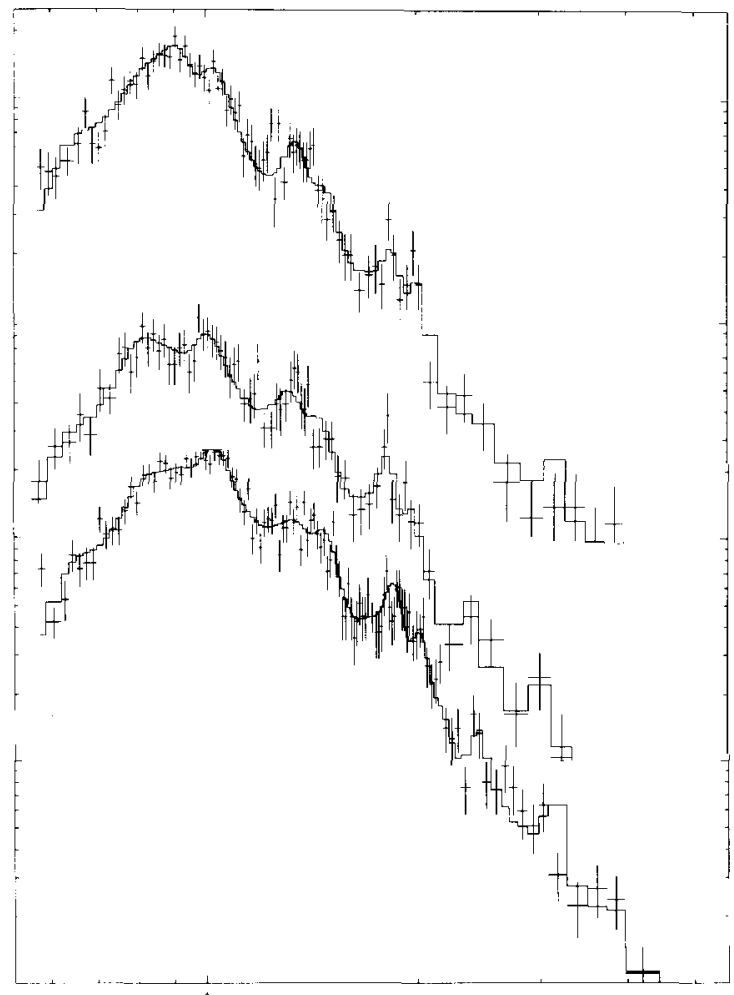

Chomnel Energy (keV)

The examples discussed above are typical of the scientific results that will come out of the BBXRT supernova remnant observations. Much more extensive analysis can be carried out on these three objects, and the other remnants will yield similar results. For instance, it will be possible to apply similar plasma diagnostics to Cas $\mathrm{A}$ as for Tycho. Also, the observation of Puppis A involved slowly scanning across the entire remnant; it will be possible to construct temperature and abundance maps of the remnant. The results from BBXRT will clearly enhance our understanding of the physical conditions in supernova remnants, and demonstrate the power of spatially resolved, high resolution spectroscopy.

\section{References}

Fahiman, G.G., and Gregory, P.C. (1981), "An X-Ray Pulsar in SNR G109.1-1.0”, Nature, 293, 202-204. Gregory, P.C., and Fahlman, G.G. (1980), “An Extraordinary New Celestial X-Ray Source”, Nature, 287, 805-807.

Hamilton, A.J.S., Sarazin, C.L., and Chevalier, R.A. (1983) "X-Ray Line Emission from Supernova Remnants. I. Models for Adiabatic Remnants", Ap. J. (Suppl.), 51, 115-148.

Hamilton, A.J.S., Sarazin, C.L., and Szymkowiak, A.E. (1986) "The X-Ray Spectrum of Tycho", Ap. J., 300, 713-721.

Morini, M., Robba, N.R., Smith, A., and van der Klis, M. (1988) "EXOSAT Observations of the Supernova Remnant G109.1-1.0 and the X-Ray Pulsar 1E 2259+589”,Ap. J., 333, 777-787.

Petre, R., Szymkowiak, A.E., Seward, F.D., and Willingale, R. (1988) "A Comprehensive Study of the XRay Structure and Spectrum of IC 443", Ap. J., 335, 215-238.

Serlemitsos, P., et al. (1991) "First Results from BBXRT", Proc. of 21st Yamada Conference, in press. 